\title{
Effect of synchronized breeding on genetic evaluations of fertility traits in dairy cattle
}

\author{
C. Lynch, ${ }^{1}$ G. A. Oliveira Junior, ${ }^{1}$ F. S. Schenkel, ${ }^{1}$ and C. F. Baes ${ }^{1,2 *}$ \\ ${ }^{1}$ Centre for Genetic Improvement of Livestock, Department of Animal Biosciences, University of Guelph, Guelph, ON, N1G 2W1, Canada \\ ${ }^{2}$ Institute of Genetics, Department of Clinical Research and Veterinary Public Health, University of Bern, Bern, 3001, Switzerland
}

\begin{abstract}
Estrus detection has become more difficult over the years due to decreases in the estrus expression of high-producing dairy cows, and increased herd sizes and animal density. Through the use of hormonal synchronization protocols, also known as timed artificial insemination (TAI) protocols, it is possible to alleviate some of the challenges associated with estrus detection. However, TAI masks cows' fertility performance, resulting in an unfair comparison of treated animals and innately fertile animals. Consequently, genetically inferior and superior cows show similar phenotypes, making it difficult to distinguish between them. As genetic programs rely on the collection of accurate phenotypic data, phenotypes collected on treated animals likely add bias to genetic evaluations. In this study, to assess the effect of TAI, the rank correlation of bulls for a given trait using only TAI records were compared with the same trait using only heat detection records. A total of 270,434 records from 192,539 animals split across heifers, first and second parity cows were analyzed for the traits: calving to first service, first service to conception, and days open. Results showed large reranking across all traits and parities between bulls compared based on either having only TAI records or only heat detection records, suggesting that a bias does indeed exist. Large reranking was also observed for both the heat detection and TAI groups among the top 100 bulls in the control group, which included all records. Furthermore, breeding method was added to the model to assess its effect on bull ranking. However, there were only minor changes in the rank correlations between scenario groups. Therefore, more complex methods to account for the apparent bias created by TAI should be investigated; for this, the method by which these data are collected needs to be improved through creating a standardized way of recording breeding codes. Though
\end{abstract}

Received March 20, 2021.

Accepted July 2, 2021

*Corresponding author: cbaes@uoguelph.ca the results of this study suggest the presence of bias within current fertility evaluations, additional research is required to confirm the findings of this study, including looking at high-reliability bulls specifically, to determine if the levels of reranking remain. Future studies should also aim to understand the potential genetic differences between the fertility traits split via management technology, possibly in a multiple-trait analysis. Key words: dairy cattle, fertility, genetic evaluations, hormonal synchronization protocols

\section{INTRODUCTION}

Reproductive goals on dairy farms revolve around increasing the rate at which eligible cows become pregnant in an optimal timely manner (Ribeiro et al., 2012). To achieve these goals, especially for AI programs, estrus detection is crucial (Roelofs et al., 2010; Silper et al., 2017). Nonetheless, estrus detection has become more difficult over the years due to decreases in the estrus expression of high-producing dairy cows caused by reduced concentrations of circulating estradiol, resulting in up to $60 \%$ of ovulations accompanied by no standing mount (Lopez et al., 2004; Wiltbank et al., 2006; Madureira et al., 2015). Also, up to $48 \%$ of cows can be anovular after the voluntary waiting period, limiting submission rates to AI after estrus detection (Santos et al., 2009). Furthermore, estrus detection can be affected by management factors such as increased herd size, animal density, time standing on concrete, and reduced quality labor, adding to the difficulty of the task (Vailes and Britt, 1990; Denis-Robichaud et al., 2016).

Through the use of hormonal synchronization protocols, also known as timed artificial insemination protocols (TAI), which synchronize follicle growth, corpus luteum regression, and ovulation, it is possible to alleviate the challenge of estrus detection, thus making ovulation time easier to predict and increasing overall pregnancy rates (Cerri et al., 2004; Ribeiro et al., 2012). The most commonly used TAI protocols stem from and include the Ovsynch protocol (Caraviello et al., 2006). 
Initially, the Ovsynch protocol achieved pregnancy rates of around 37\% (Pursley et al., 1997), but more recently variations have been developed with commercial success including Presynch-Ovsynch, Double-Ovsynch, and PRID-Synch (Moreira et al., 2001; Souza et al., 2008; Ribeiro et al., 2011; Santos et al., 2016). These advancements have allowed pregnancy rates to reach between $40 \%$ and $50 \%$ in high-producing dairy cows (Santos et al., 2017).

The effect of TAI in terms of reproductive and economic improvements is more pronounced on farms with low $(<50 \%)$ estrus detection (Leblanc, 2001; Tenhagen et al., 2004). The combined control of first insemination, ability to improve fertility of anovular cows, overall higher pregnancy rates, and economic implications have increased the uptake of TAI (Ribeiro et al., 2012). In Canada, TAI is routinely used, with the Canadian Dairy Network (CDN) reporting that in 2017 approximately $30 \%$ of herds had more than $50 \%$ of their breeding done on 1 or $2 \mathrm{~d}$ of the week, suggesting the use of TAI (Van Doormaal, 2018). This number has continually increased over the past 2 decades and shows no signs of slowing down. In the United States, the usage of TAI is even higher, where $87 \%$ of herds were estimated to be using TAI (Caraviello et al., 2006).

Though TAI has proven to be a successful management tool in dairy cattle, there are potential issues with TAI from a genetic breeding program point of view. Timed AI affects the reproductive physiology and endocrinology involved in the estrous cycle, allowing for higher pregnancy rates. Timed AI masks cows' fertility performance, resulting in an unfair comparison of treated animals and innately fertile animals (Fleming et al., 2018). Consequently, genetically inferior and superior cows show similar phenotypes, making it difficult to distinguish between them. As genetic programs rely on the collection of accurate phenotypic data, phenotypes collected on treated animals likely add bias to genetic evaluations (Tsuruta et al., 2000; Bouquet and Juga, 2013). A recent simulation study by Oliveira Junior et al. (2021) looked at multiple parameters to indirectly measure bias when using TAI records on genetic evaluations of fertility traits. The results of the study found that as the usage rate of TAI increased, there were unfavorable changes in all analyzed parameters for female reproductive traits.

In the current study, real data provided by 2 Lactanet partners, CanWest DHI and CDN, were used to verify the results found in the simulation by Oliveira Junior et al. (2021) and to better understand the real effects of TAI protocols on genetic evaluations. To assess the effect of TAI, changes in the rank correlation of bulls for a trait using only TAI records were compared with the same trait using only heat detection records. If no bias existed, we would expect bulls to rank the same in both situations. However, if there is large reranking, it is highly likely that TAI is causing a bias in genetic evaluations of fertility traits, thus reducing the accuracy of the evaluations. A control scenario was also added whereby all records were included regardless of breeding method. The top 100 bulls from the control scenario were compared against both the heat detection and TAI groups to identify the effect of TAI on highgenetic-merit bulls under the current system. Furthermore, breeding method was added to an updated model to assess its capacity for compensating against any potential bias reported in the initial analyses. Overall, the objective of this study was to assess the potential bias that TAI might add to the estimated genetic parameters and breeding values of female fertility traits.

\section{MATERIALS AND METHODS}

\section{Breeding Data}

Breeding data provided by Lactanet consisted of 4,420,685 Canadian Holstein service records ranging from lactations 1 to 14 for 777,116 cows across 1,192 herds from 2009 to 2019. There was a total of 5,804 breeding codes across the breeding records, of which 2,046 were unique. Breeding codes are single-letter codes that correspond to a breeding protocol description and are unique for each herd. Of these 2,046 unique codes, 65 codes had a frequency of 10 or more, 312 had a frequency of 2 to 9 , and 1,668 codes had a frequency of 1 , meaning they only occurred on a single herd. The reason behind this wide range of breeding codes is that producers define the breeding codes themselves, leading to numerous ways of recording the same breeding method. Due to the wide range of breeding protocols, breeding codes were classified into 2 scenario groups for comparison. A protocol was classified as fixed-time AI (FTAI) if it was a clear TAI protocol and as HD if it was a clear heat-detection protocol. A control scenario was also added, which included all available records regardless of protocol. In general, the control groups were split $67 \%$ HD records and 33\% FTAI records. Clear TAI protocols included protocols that involved a hormone treatment cycle with a predetermined insemination time, and therefore, did not include simple hormone treatments such as a shot of $\mathrm{GnRH}$, which can be used as a treatment for cystic ovaries. Clear heat detection protocols included protocols where animals were in control of expressing heat, and included several conventional methods such as standing heat, as well as more advanced methods, for example milk progesterone levels. Protocols that did not fall into either of these scenarios were left unclassified, and included numerous 
obscure protocols and protocols in which hormones were used as treatments for sick animals. Animals with both FTAI and HD records within the same parity had these records removed. This crossover occurred when animals had multiple services within a parity where producers used various techniques to get the cow pregnant. Of the original 5,804 breeding codes, these classifications resulted in 2,002 protocols classified as FTAI, whereas a further 1,840 were classified as HD. The remaining 1,962 codes were unclassified and removed from the analysis.

\section{Merged Data}

Upon classification of the 3 scenario groups (FTAI, $\mathrm{HD}$, and control), the corresponding fertility traits information was merged with the breeding data. Fertility data provided by Lactanet composed of 1,165,871 records from 563,817 Holstein cows. Three fertility traits, thought to be the most affected by TAI were selected: (1) calving to first service (CTFS), defined as the number of days between calving and first insemination; (2) first service to conception (FSTC), defined as the number of days between the first insemination and the insemination that results in a calf; and (3) days open (DO), defined as the number of days from calving to conception, which equals the summation of CTFS and FSTC.

Parities were considered separately, with records from parity 0 (heifers), parity 1 , and parity 2 used in the analysis. For quality control, trait values that did not match between the breeding and fertility data sets were removed. Records where CTFS + FSTC $\neq$ DO were removed. This generally occurred where cows had a late term abortion, as in this case, FSTC restarts from the first service post-abortion. Any cows with a confirmed calving missing any of the 3 fertility trait phenotypes were also removed. Open cows only required a CTFS value, whereas heifers only required a FSTC value. Contemporary groups (CG) with fewer than 5 animals were also removed. Last, connectedness of CG was analyzed using the AMC program and disconnected CG were removed (Roso et al., 2004). The merged data resulted in a total of 270,434 records from 192,539 cows. The final data sets used in the analysis are shown in Table 1.

\section{Statistical Analysis}

Descriptive statistics were calculated using R statistical software version 3.5.1 (R Core Development Team, 2019). The Lilliefors (Kolmogorov-Smirnov) single variable normality test in $\mathrm{R}$ was used to determine the normality of each trait distribution. The REML pro- cedure in ASReml 4.1 (Gilmour et al., 2014) was used to estimate variance components and predict breeding values (EBVs) for the animals in each scenario group. Pedigree files containing 8 generations for animals of each parity and scenario group were created. Upon convergence of the mixed model equations, $\mathrm{R}$ was used to calculate model-based reliabilities and determine the rank correlations of both the reliabilities and EBVs. In $\mathrm{R}$, animals were ranked using the function rank within the base package and correlations were determined by using the cor.test function within the stats package ( $\mathrm{R}$ Core Development Team, 2019). The reliability rank correlations were calculated to account for the differences in mean reliabilities of common bulls across scenario groups. Reliabilities were calculated using the method presented by Van Vleck (1993) assuming no inbreeding:

$$
\text { Reliability }=1-\frac{P E V_{i}}{\sigma_{a}^{2}},
$$

whereby $\sigma_{a}^{2}$ is the additive genetic variance and $P E V_{i}$ is the prediction error variance of the $i$ th animal.

\section{Models}

Univariate animal models were used to estimate genetic parameters and predict EBVs for all traits. The models used in this study were adaptions of those used by Lactanet in their genetic evaluations for fertility traits (Jamrozik et al., 2005). Model parameters are as follows:

$$
y_{i j k l}=\mu+R Y M_{i}+A M_{j}+H_{k}+a_{l}+e_{i j k l}
$$

where $y_{i j k l}$ is the observed phenotype (CTFS, FSTC, or DO) of the lth cow; $R Y M_{i}$ is the fixed effect of regionyear-month born; $A M_{j}$ is the fixed effect of age previous calving-month previous calving for DO and CTFS, whereas for FSTC, $A M_{j}$ is the fixed effect of age previous calving-month first service; $H_{k}$ is the fixed effect of herd in the original analyses, whereas in the updated

Table 1. Total number of records for each scenario group by parity

\begin{tabular}{lrcc}
\hline & \multicolumn{3}{c}{ Records (herds) } \\
\cline { 2 - 4 } Item $^{1}$ & Parity 0 & Parity 1 & Parity 2 \\
\hline FTAI & $9,068(211)$ & $33,038(475)$ & $22,479(443)$ \\
HD & $101,231(659)$ & $64,330(637)$ & $40,288(593)$ \\
Control & $110,299(675)$ & $97,368(698)$ & $62,767(660)$ \\
\hline
\end{tabular}

${ }^{1} \mathrm{FTAI}=$ fixed-time $\mathrm{AI}$ protocol; $\mathrm{HD}=$ heat detection protocol. 
Table 2. Preliminary statistics for calving to first service (CTFS), first service to conception (FSTC), and days open $(\mathrm{DO})^{1}$

\begin{tabular}{llccccc}
\hline \multirow{2}{*}{ Trait } & $\begin{array}{l}\text { Scenario } \\
\text { group }\end{array}$ & $\mathrm{n}$ & Median & MAD & Kurtosis & LT D-score \\
\hline CTFS & FTAI & 55,517 & 74 & 10.38 & 11.49 & 0.14 \\
& HD & 104,618 & 71 & 17.79 & 6.39 & 0.13 \\
& Control & 160,135 & 73 & 16.31 & 7.70 & 0.13 \\
& FTAI & 56,257 & 1 & 0 & 5.46 & 0.44 \\
& HD & 190,367 & 1 & 0 & 6.20 & 0.36 \\
& Control & 246,624 & 1 & 0 & 6.03 & 0.38 \\
& FTAI & 47,189 & 82 & 20.76 & 3.58 & 0.18 \\
& HD & 89,136 & 86 & 32.62 & 2.69 & 0.13 \\
& Control & 136,325 & 84 & 28.17 & 2.99 & 0.15 \\
\hline
\end{tabular}

${ }^{1} \mathrm{n}=$ number of records; MAD $=$ median absolute deviation; $\mathrm{LT}=$ Lilliefors (Kolmogorov-Smirnov) normality test. FTAI = fixed-time AI protocol; HD = heat detection protocol.

model, $H_{k}$ is the fixed effect of herd-breeding method; $a_{l}$ is the additive genetic effect of the $l$ th cow; and $e_{i j k l}$ is the vector of random residuals. The covariance matrix $(\mathbf{V})$ was defined as

$$
\mathbf{V}=\operatorname{var}\left[\begin{array}{l}
a \\
e
\end{array}\right]=\left[\begin{array}{cc}
\mathbf{A} \sigma_{a}^{2} & 0 \\
0 & \mathbf{I} \sigma_{e}^{2}
\end{array}\right],
$$

assuming that $\left[\begin{array}{l}a \\ e\end{array}\right] \sim N(0, \mathbf{V})$, where $\mathbf{A}$ is the additive relationship matrix, $\mathbf{I}$ is an identity matrix, $\sigma_{a}^{2}$ is the additive genetic variance and $\sigma_{e}^{2}$ is the residual variance.

\section{RESULTS AND DISCUSSION}

\section{Descriptive Statistics}

Descriptive statistics for CTFS, FSTC, and DO in each scenario group are presented in Table 2. For the purpose of this study, only the FTAI and HD values will be discussed, but in all cases, the control scenario showed intermediate values between the 2 scenario groups, which was expected as it included all records. The Lilliefors (based on Kolmogorov-Smirnov) single variable normality test was applied to help better understand the descriptive statistics. For FSTC, D-scores of 0.44 and 0.36 were obtained indicating that there are large differences between the normal distribution and both the FTAI and the HD FSTC distributions. Therefore, the preliminary statistics of FSTC are less insightful compared with both CTFS and DO, which produced D-scores below 0.18 , indicating that the distributions did not largely deviate from normal. Due to the positive skewness of the CTFS and DO distributions, the median and median absolute devia- tion (MAD) are presented instead of the mean and standard deviation. To highlight each trait's distribution, density distribution graphs for each trait in both scenario groups are shown in Figure 1. Furthermore, it is important to note that in each trait, the median values are smaller than those observed in the population. This occurred because by removing animals exposed to both HD and FTAI protocols within the same parity, many poor performing animals were removed, resulting in the median value decreasing.

There were lower MAD values and larger kurtosis values in the FTAI group compared with the HD group, and this reduced variation is evident from the density distributions in Figure 1. In Figure 1A for CTFS, there is a more evident peak around $75 \mathrm{~d}$ in the FTAI group compared with an even distribution in the HD group. CTFS is a trait highly influenced by management, especially when using hormone protocols. In this case, a producer is in complete control of when the animal cycles and when the animal is serviced, resulting in more consistent results. In the case of heat detection, the animal's natural metabolic functions are in control of when they cycle, leading to more variation within the population. When comparing the median values of CTFS, heat detection protocols showed lower values than TAI protocols. A possible explanation is that in mixed management herds, TAI is more frequently used on cows with delayed or unobserved estrus (Goodling et al., 2005; Ribeiro et al., 2012). Furthermore, animals on TAI protocols that show estrus before the target day are often bred on natural heat (Goodling et al., 2005). To investigate, TAI herds were identified with less than $10 \%$ TAI records. This equated to $25 \%$ of the total herds in the data set, meaning that a large number of herds are potentially using TAI as a tool for infertile animals. Together, these factors contribute to the reduced variation and larger median values seen in TAI animals for CTFS. 
Figure 1B shows the density distribution of FSTC, and although most values center around 1 , there are multimodal distributions for each scenario group past the initial spike. In the HD group, there are 2 other spikes, at around 21 and $42 \mathrm{~d}$, representing a cow's estrous cycle. However, in FTAI, these 2 spikes occurred around 40 and $80 \mathrm{~d}$, which varies from the traditional 21-d estrous cycle. A possible explanation is that cows on TAI are usually checked roughly 30 d postinsemination to confirm if they are pregnant. Nonpregnant cows are then subjected to a TAI protocol again, usually taking around a week to $10 \mathrm{~d}$ until breeding, thus explaining the spikes in the data around $40 \mathrm{~d}$ apart.
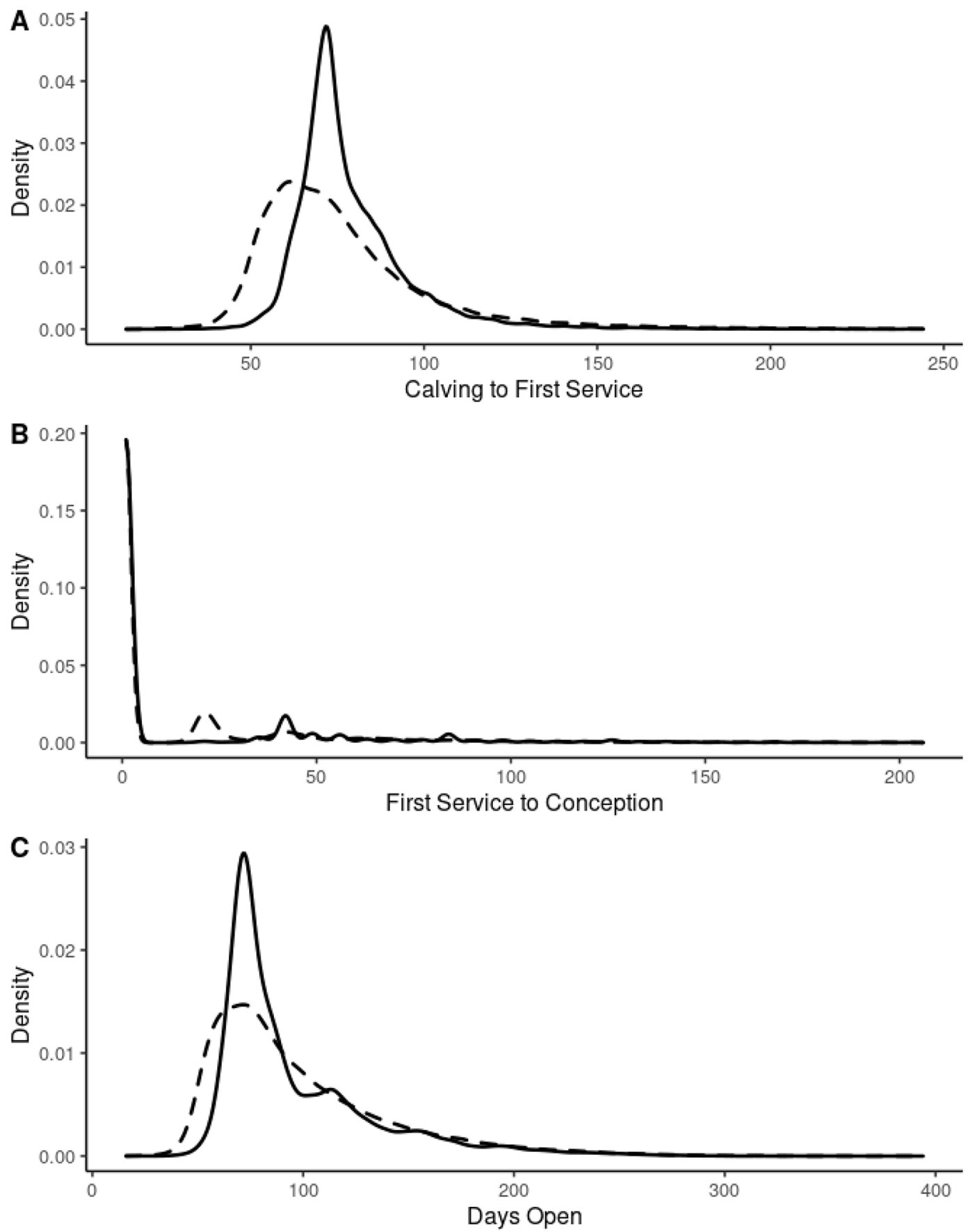

Figure 1. Density distributions for calving to first service (A), first service to conception (B), and days open (C) (timed AI protocol = whole line; heat detection protocol $=$ dashed line). 
Table 3. Variance components and heritability $\left(\mathrm{h}^{2}\right)$ estimates (SE in parentheses) ${ }^{1}$

\begin{tabular}{|c|c|c|c|c|}
\hline \multirow[b]{2}{*}{ Trait } & \multirow[b]{2}{*}{$\begin{array}{l}\text { Scenario } \\
\text { group }\end{array}$} & \multicolumn{3}{|c|}{ Variance component } \\
\hline & & $\begin{array}{l}\text { Phenotypic } \\
\text { variance }\end{array}$ & $\begin{array}{c}\text { Genetic } \\
\text { variance }\end{array}$ & $h^{2}$ \\
\hline \multirow[t]{6}{*}{ CTFS } & FTAI 1 & $187.4(1.5)$ & $4.9(1.5)$ & $0.026(0.008)$ \\
\hline & HD 1 & $469.0(2.7)$ & $19.0(3.0)$ & $0.041(0.006)$ \\
\hline & Control 1 & $399.7(1.9)$ & $21.2(2.4)$ & $0.053(0.006)$ \\
\hline & FTAI 2 & $177.1(1.7)$ & $2.8(1.2)$ & $0.016(0.007)$ \\
\hline & HD 2 & $485.9(3.5)$ & $17.0(3.3)$ & $0.035(0.007)$ \\
\hline & Control 2 & $404.2(2.4)$ & $15.4(2.4)$ & $0.038(0.006)$ \\
\hline \multirow[t]{9}{*}{ FSTC } & FTAI 0 & $731.2(11.2)$ & $0.0001(0.000002)$ & $0.000(0.000)$ \\
\hline & HD 0 & $622.9(2.8)$ & $4.1(1.3)$ & $0.008(0.002)$ \\
\hline & Control 0 & $635.3(2.7)$ & $3.8(1.2)$ & $0.006(0.002)$ \\
\hline & FTAI 1 & $1,070.0(9.1)$ & $19.0(6.6)$ & $0.019(0.006)$ \\
\hline & HD 1 & $1,110.4(6.7)$ & $18.6(4.6)$ & $0.017(0.004)$ \\
\hline & Control 1 & $1,109.1(5.5)$ & $20.6(3.9)$ & $0.019(0.004)$ \\
\hline & FTAI 2 & $1,148.6(12.3)$ & $29.5(10.5)$ & $0.026(0.009)$ \\
\hline & HD 2 & $1,293.2(10.4)$ & $40.1(9.5)$ & $0.031(0.007)$ \\
\hline & Control 2 & $1,249.7(8.0)$ & $30.2(6.5)$ & $0.024(0.005)$ \\
\hline \multirow[t]{6}{*}{ DO } & FTAI 1 & $1,241.3(10.6)$ & $31.1(8.9)$ & $0.025(0.007)$ \\
\hline & HD 1 & $1,520.7(9.3)$ & $42.0(8.1)$ & $0.028(0.005)$ \\
\hline & Control 1 & $1,452.7(7.3)$ & $45.7(6.6)$ & $0.032(0.005)$ \\
\hline & FTAI 2 & $1,305.4(14.0)$ & $45.1(14.0)$ & $0.035(0.010)$ \\
\hline & HD 2 & $1,670.9(13.4)$ & $61.3(13.0)$ & $0.037(0.008)$ \\
\hline & Control 2 & $1,559.7(10.0)$ & $52.2(9.3)$ & $0.033(0.006)$ \\
\hline
\end{tabular}

$\overline{{ }^{1} \mathrm{CTFS}}=$ calving to first service $(\mathrm{d}) ; \mathrm{FSTC}=$ first service to conception $(\mathrm{d}) ; \mathrm{DO}=$ days open $(\mathrm{d}) ; 0,1$, and 2 $=$ parity number. $\mathrm{FTAI}=$ fixed-time $\mathrm{AI}$ protocol; $\mathrm{HD}=$ heat detection protocol.

Last, Figure 1C shows the density distribution of DO. The FTAI group produced clearer spikes in the distribution in comparison with $\mathrm{HD}$, which presented a smoother distribution. This results from a combination of the 2 previously discussed distributions (CTFS and FSTC), which make up DO. Overall, these distributions have differences in variation due to the level of management involved in each trait. The values for each trait represent the expected values for the 3 traits, meaning that the majority of breeding codes were split into scenario groups correctly, therefore making this data set appropriate for the analysis.

\section{Variance Components}

Variance components for each scenario group are presented in Table 3, which includes a control scenario for each trait. The control scenario contains all records, and therefore, better represents current evaluations, as breeding protocol is disregarded. Given this, the differences between the control and HD groups provides the best indication to the level of bias present in current genetic evaluations. However, differences between the control and HD are specific to this study and should not be considered the true effect of TAI on current genetic evaluations. This is because differences will be dictated by the number of records a bull has on cows that used either TAI or heat detection, which was roughly split $67 \%$ heat detection records and $33 \%$ TAI records within this study. This split is specific to this study and may not be representative of other evaluations. Therefore, given that the objective of this initial study was to identify if a bias exists when using TAI records, as opposed to estimating the extent of the bias, 2 extreme scenarios, where a bull either has $100 \%$ TAI records (FTAI) or $100 \%$ heat detection records (HD), were compared.

The observed heritability estimates for CTFS, FSTC, and DO were similar within traits across parities and to published values (Kadarmideen et al., 2003; Jamrozik et al., 2005; Pritchard et al., 2013). However, there was a trend of reduced phenotypic and genetic variances across all traits in the FTAI group. The most apparent differences in variance between FTAI and HD were exhibited in CTFS, with significant differences between phenotypic and genetic variance estimates of both scenario groups. As previously discussed, this reduced variation was expected due to the influence management has on CTFS when using TAI. Results agree with previous work of Lucy et al. (1986), who found reduced phenotypic variation in CTFS for animals using TAI compared with heat detection.

For FSTC in parity 1 and 2 cows, reduced phenotypic variation in the FTAI group was observed, whereas the estimated genetic variances were not different when accounting for standard errors. Interestingly, in heif- 
ers, larger phenotypic variation for FTAI compared with HD was observed. This larger variation may be due to the limited sample size within FTAI for heifers but may also be linked to the limited success rate of hormonal protocols in heifers. Numerous TAI protocols have limitations for heifers, mainly because up to $50 \%$ of heifers have estrous cycles with 3 or more follicular cycles (Sartori et al., 2004). More follicular cycles limit the time a dominant follicle is active and responsive to LH-induced ovulation, causing asynchrony with expressed estrus before TAI (Rivera et al., 2005; Bisinotto and Santos, 2011). Heifers also have larger progesterone concentrations than lactating cows, which blocks the GnRH-induced luteinizing hormone release that might impair ovulation (Giordano et al., 2012; Lima et al., 2013; Madureira et al., 2021). These issues could also explain estimated genetic variance for FSTC in heifers, which was close to zero, meaning the model was struggling to estimate the genetic influence in heifers for FSTC. New protocols are being designed for heifers, mainly revolving around a 5-d TAI, with numerous studies exploring successful variations around this base (Rabaglino et al., 2010; Karakaya-Bilen et al., 2019). With continued improvements in TAI designed for heifers, we will likely see an increase in the number of records and a reduction in the phenotypic variation.

One issue with the scenario groups, specifically for estimating genetic parameters of FSTC, was that by editing out animals who were under both TAI and heat detection within a given parity, we inherently selected the more fertile animals within the sample. Most animals that were removed had a higher number of services on average, which is expected as producers were using multiple techniques to get the cows pregnant. This step affects the FTAI group more, as producers often use TAI on animals struggling to get pregnant, and this may partially explain the reduced phenotypic variance within the FTAI group across traits (Goodling et al., 2005; Ribeiro et al., 2012). However, this issue should not affect the results of the bulls' rank correlations, as bulls still required records from both scenario groups, which are under the same constraints. This quality control factor was crucial as the impact of TAI could be better determined, since animals were not exposed to any other breeding methods within a parity. Last, DO followed a similar trend with reduced phenotypic and genetic variance estimates seen in the FTAI group. However, once again when accounting for the standard errors the estimated genetic variance was not different across parities. As DO is a function of both CTFS and FSTC, their respective results profoundly influence DO and explain the intermediate relative differences of DO compared with CTFS and FSTC.

\section{Rank Correlations}

The EBV rank correlations, together with the reliability rank correlations of common bulls for the scenario groups, are presented in Table 4. Again, as the purpose of the initial analysis was to identify if a bias exists when using TAI records, only the FTAI and HD scenarios were compared. Reliability rank correlations were calculated to account for the differences in mean reliabilities, due to unbalanced records of common bulls across scenario groups (Table 5). When making inferences on the EBV rank correlations, a high-reliability rank correlation of bulls across scenarios would indicate that the EBV rank correlations would not change due to the amount of information (i.e., number of daughters) across scenarios. In the case of a low-reliability rank correlation, bulls may be simply changing EBV rank due to having more information in one scenario compared with another, as EBVs are shrunk toward zero with limited information. Nearly all reliability rank correlations were above 0.9 , suggesting that changes in the EBV rank were not due to the relative difference in record counts between the bulls across groups. The FSTC in heifers was the only trait that did not follow this trend and had a reliability rank correlation of 0.05 , which can be explained by the mean reliability value of near zero for bulls in the FTAI group. The reasoning behind such a low correlation can be partially attributed to the imbalance of records in this comparison group. As previously mentioned, there are challenges with TAI in heifers, and this may also help explain the reduced reliability of bulls in this scenario group. Bulls had low reliabilities because their genetic effect was potentially masked by the inefficiency of specific TAI protocols in heifers, making it difficult for the model to predict EBVs with confidence. This point is further justified through the estimated genetic variance in heifers for FSTC, in the FTAI group. Here the genetic variance estimate was close to zero, meaning the model was unable to identify the genetic effect component of the trait. It is likely that a genetic effect on FSTC in heifers exists; therefore, further analysis considering the specific TAI protocol used for these bulls would help to better understand the results.

The EBV rank correlations of the common bulls for CTFS showed a correlation of 0.40 and 0.38 between FTAI and HD for parity 1 and 2, respectively. Though these are moderate correlations, considerable reranking is clearly occurring. It is possible that reranking may be occurring due to the low-reliability values presented in Table 5. However, it is unlikely that this fully explains the reported levels of reranking. Even more apparent differences were observed when FSTC was compared 
Table 4. Reliability (upper diagonal) and EBV (below diagonal) rank correlation of common bulls between scenario groups ${ }^{1}$

\begin{tabular}{llclc}
\hline \multirow{2}{*}{ Trait } & $\begin{array}{l}\text { Scenario } \\
\text { group }\end{array}$ & FTAI & HD & Control \\
\hline CTFS & FTAI 1 & - & 0.90 & 0.85 \\
& HD 1 & 0.40 & - & 0.96 \\
& Control 1 & 0.58 & 0.92 & - \\
& FTAI 2 & $\overline{-}$ & 0.92 & 0.90 \\
FSTC & HD 2 & 0.38 & - & 0.97 \\
& Control 2 & 0.57 & 0.93 & - \\
& FTAI 0 & - & 0.05 & 0.05 \\
& HD 0 & -0.06 & - & 1.00 \\
& Control 0 & 0.21 & 0.93 & - \\
& FTAI 1 & - & 0.96 & 0.96 \\
& HD 1 & 0.17 & - & 0.98 \\
Control 1 & 0.57 & 0.86 & - \\
& FTAI 2 & - & 0.95 & 0.96 \\
& HD 2 & 0.17 & - & 0.99 \\
& Control 2 & 0.54 & 0.88 & - \\
& FTAI 1 & - & 0.94 & 0.93 \\
& HD 1 & 0.45 & - & 0.97 \\
& Control 1 & 0.71 & 0.91 & - \\
& FTAI 2 & - & 0.94 & 0.96 \\
& HD 2 & 0.38 & - & 0.98 \\
& Control 2 & 0.69 & 0.89 & - \\
\hline
\end{tabular}

${ }^{1}$ CTFS $=$ calving to first service $(\mathrm{d}) ; \mathrm{FSTC}=$ first service to conception $(\mathrm{d}) ; \mathrm{DO}=$ days open $(\mathrm{d}) ; 0,1$, and $2=$ parity number. FTAI $=$ fixed-time AI protocol; HD = heat detection protocol.

between the FTAI and HD groups, with EBV rank correlations of $-0.06,0.17$, and 0.17 for heifers, parity 1 , and parity 2 cows, respectively. As previously mentioned, heifer results had a low-reliability rank correlation, and therefore some reranking is likely due to differences in record count between the scenario groups. However, this low-reliability rank correlation likely does not fully explain the negative EBV correlation between the traits, which is also likely due to differences in model prediction. Results for both parity 1 and 2 animals support this as their EBV rank correlations showed a minimal relationship between the traits while having very strong reliability rank correlations of 0.96 and 0.95 , respectively. This level of reranking indicates that FSTC measured in FTAI and HD are likely 2 completely different traits. Days open, like CTFS, produced moderate EBV rank correlations of 0.45 and 0.38 for parity 1 and 2 animals, respectively, coupled with highreliability rank correlations of 0.94 in both parities.

Overall, it was evident in all 3 traits analyzed that large reranking occurred when bulls had only TAI records as opposed to only heat detection records. However, it is essential to note that in all cases, the control groups showed much less reranking when compared against $\mathrm{HD}$, and this change in reranking better represents the potential bias present in practice in the current genetic evaluations in Canada. As previously mentioned, the differences between the control and HD groups are dictated by the split of TAI and heat detection records within this study, which is liable to change substantially in the future in this population, and in other populations. The use of TAI is continuingly increasing in Canada, and this has been made further evident from the analyzed data. Figure 2 illustrates the changes in TAI usage in both animals and herds between 2009 and 2019 within the data set. Between these years, the percentage of herds using TAI has increased from $30 \%$ to $59 \%$, whereas the percentage of animals using TAI increased from $12 \%$ to nearly $18 \%$. Therefore, the effect of TAI on genetic evaluations of fertility traits will likely continue to grow under the current system. Values here differ from those published by CDN, whereby they estimated the number of herds using TAI by assuming that any herds with more than $50 \%$ of their breedings on 1 or $2 \mathrm{~d}$ of the week to be using TAI, to which they estimated $30 \%$ of herds as of 2017 . Within these data, it was shown to be nearly $60 \%$, and the reason behind this is likely because it is common for producers to identify problem cows before breeding and assign them to TAI (Goodling et al., 2005). As previously mentioned, TAI herds were identified with less than $10 \%$ TAI records, which equates to $25 \%$ of the total herds in the data set, and this fact explains the

Table 5. Mean reliabilities of common bulls between scenario groups ${ }^{1}$

\begin{tabular}{|c|c|c|c|c|c|c|}
\hline \multirow[b]{2}{*}{ Parity } & \multirow{2}{*}{$\begin{array}{l}\text { Scenario } \\
\text { group }\end{array}$} & \multirow[b]{2}{*}{ Total bulls } & \multirow[b]{2}{*}{ Records } & \multicolumn{3}{|c|}{ Mean reliability } \\
\hline & & & & CTFS & FSTC & DO \\
\hline \multirow[t]{3}{*}{0} & FTAI & 1,592 & 8,451 & - & 0 & - \\
\hline & $\mathrm{HD}$ & & 73,321 & - & 0.21 & - \\
\hline & Control & & 81,772 & - & 0.21 & - \\
\hline \multirow[t]{3}{*}{1} & FTAI & 3,327 & 29,368 & 0.22 & 0.17 & 0.20 \\
\hline & $\mathrm{HD}$ & & 55,218 & 0.33 & 0.23 & 0.27 \\
\hline & Control & & 84,586 & 0.40 & 0.28 & 0.33 \\
\hline \multirow[t]{3}{*}{2} & FTAI & 2,753 & 19,522 & 0.14 & 0.17 & 0.20 \\
\hline & HD & & 33,768 & 0.28 & 0.24 & 0.26 \\
\hline & Control & & 53,290 & 0.33 & 0.27 & 0.30 \\
\hline
\end{tabular}

${ }^{1}$ CTFS $=$ calving to first service $(d) ;$ FSTC $=$ first service to conception $(d) ;$ DO $=$ days open $(d)$. FTAI $=$ fixed-time AI protocol; $\mathrm{HD}=$ heat detection protocol. 


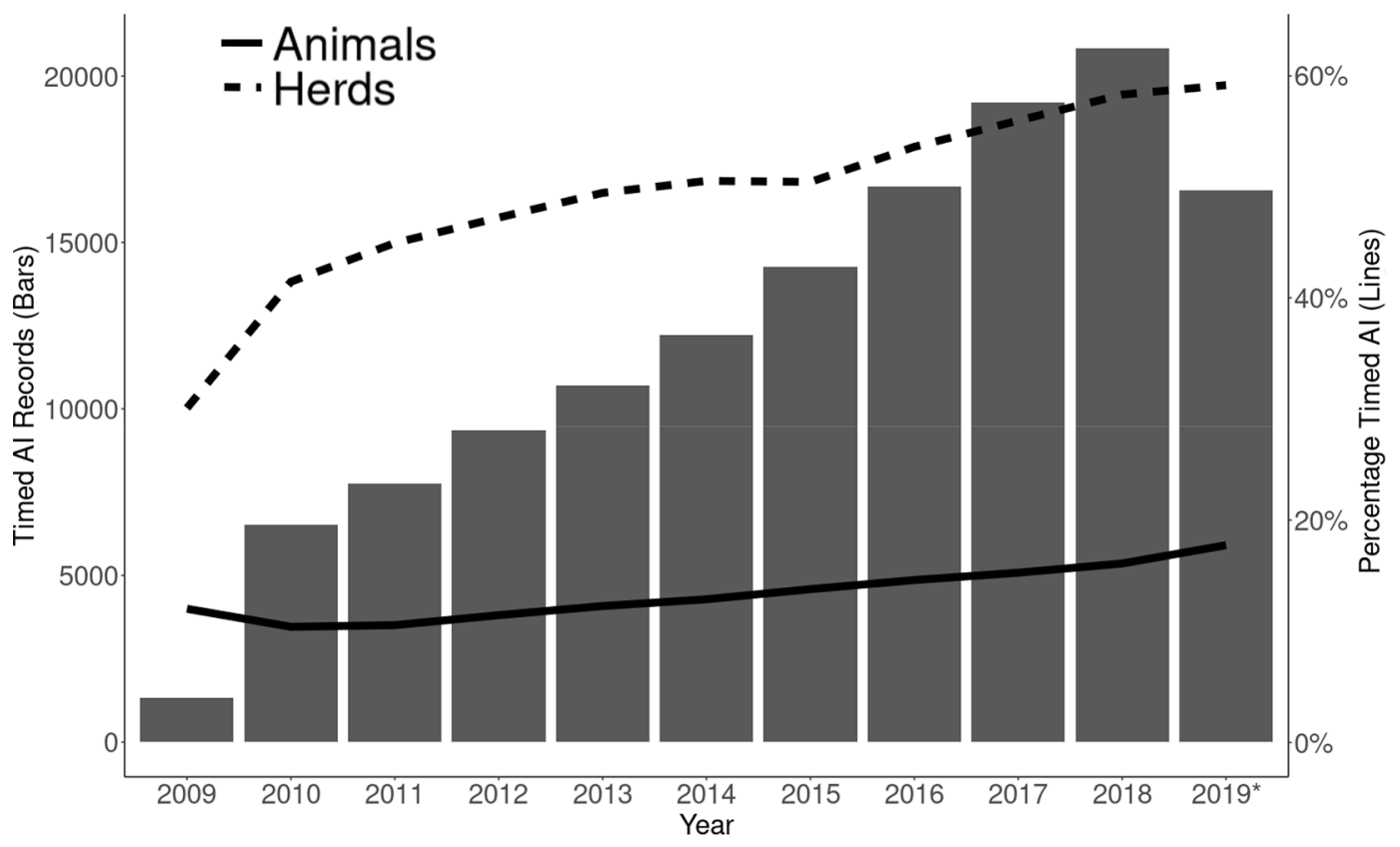

Figure 2. Changes in timed AI usage rate in animals and herds between 2009 and 2019 on Canadian herds that are members of DHI DairyComp. Note: records from 2019 are incomplete.

difference between the current study and the previous study done by CDN. This fact further highlights the issue of TAI for genetic evaluations, as these infertile animals are becoming hidden and performing similarly to naturally fertile animals, emphasized by the very similar median values in each of the 3 traits (Table 1 ).

Overall, additional analysis is required to quantify the potential bias that exists when using TAI records and how to account for it in genetic analysis. Alternatively, these traits should be treated as 2 separate traits within selection indices and be analyzed by a multiple trait model. To confirm the findings of this study, future research should focus on estimating the genetic correlation between these traits to better understand their potential genetic dissimilarity. Furthermore, bulls with a high reliability should be looked at specifically to determine if the levels of reranking remain. Unfortunately, the current study was unable to investigate this because there were limited bulls in both scenario groups with high reliabilities.

\section{Effect on Top Bulls}

To investigate the effect of TAI on high genetic merit bulls, the rank correlations of the top 100 bulls from the control scenario were compared against both the HD and FTAI groups (Table 6). The main takeaway was the reduced EBV rank correlations between both scenario groups and the control scenario group compared with the original full analysis, whereas the re- liability rank correlation remained stable across both analyses. Across traits, correlations decreased by an average of 0.34 and 0.41 for the HD and FTAI groups, respectively. Furthermore, the average proportional reduction in rank correlation was $70.2 \%(38.9-91.4 \%)$ across traits and parities for the FTAI group, which was much higher than $37.5 \%$ (24.7-48.8\%) observed for the HD group. This stronger reranking for the FTAI group among the 100 top control bulls compared with the HD group suggests that top performing bulls are not predicted well when only TAI records are considered. The reasoning behind this is unclear, but may be due to the reduced variation in performance with TAI, which may affect the model's capacity to clearly identify bulls with high fertility performance daughters.

The reasoning behind the drop in each correlation is due to the fact that only extreme values were looked at, as only the top 100 bulls were examined. When only looking at extremes of a normal distribution, the range and variation of the variables are reduced, and therefore, the linear relationship between them (linear correlation) tends to decrease as well (Goodwin and Leech, 2006). Though this explains the decrease in correlations, it does not take away from the importance of this analysis. In the current system, these high-performance bulls are used most commonly and therefore are of most interest. Therefore, this analysis better represents the expected correlations that would be seen if this work was undertaken on any national selection index, as only high-performance bulls in relation to the population are 
Table 6. Reliability and EBV rank correlations between the top 100 bulls in the control scenario and both the fixed-time AI (FTAI) and heat detection (HD) scenario groups ${ }^{1}$

\begin{tabular}{llcc}
\hline & & \multicolumn{2}{c}{ Control rank correlation } \\
\cline { 2 - 4 } Trait & $\begin{array}{l}\text { Scenario } \\
\text { group }\end{array}$ & EBV & Reliability \\
\hline CTFS & FTAI 1 & 0.05 & 0.87 \\
& HD 1 & 0.51 & 0.96 \\
& FTAI 2 & 0.06 & 0.87 \\
FSTC & HD 2 & 0.62 & 0.98 \\
& FTAI 0 & 0.11 & 0.19 \\
& HD 0 & 0.66 & 1 \\
& FTAI 1 & 0.23 & 0.95 \\
& HD 1 & 0.45 & 0.98 \\
\multirow{2}{*}{ DO } & FTAI 2 & 0.33 & 0.96 \\
& HD 2 & 0.43 & 0.99 \\
& FTAI 1 & 0.12 & 0.95 \\
& HD 1 & 0.59 & 0.98 \\
& FTAI 2 & 0.13 & 0.95 \\
& HD 2 & 0.67 & 0.99 \\
\hline
\end{tabular}

${ }^{1}$ CTFS $=$ calving to first service $(\mathrm{d}) ;$ FSTC $=$ first service to conception $(\mathrm{d}) ; \mathrm{DO}=$ days open $(\mathrm{d}) ; 0,1$, and $2=$ parity number.

of interest. Overall, this analysis emphasizes the need for further investigation into quantifying the effect of TAI records on genetic evaluations of fertility traits, as differences appear to be maximized when looking directly at elite bulls.

\section{Updated Model}

To investigate methods to account for the apparent bias seen by the inclusion of TAI records within genetic evaluations of fertility traits, breeding method was added to the model as a fixed effect. The updated model included a herd-breeding method factor as opposed to

Table 7. Reliability and EBV rank correlations between the control scenario and both the fixed-time AI (FTAI) and heat detection (HD) scenario groups for common bulls between groups when breeding method added to the model ${ }^{1}$

\begin{tabular}{llcc}
\hline & & \multicolumn{2}{c}{ Control rank correlation } \\
\cline { 3 - 4 } Trait & $\begin{array}{l}\text { Scenario } \\
\text { group }\end{array}$ & EBV & Reliability \\
\hline CTFS & FTAI 1 & 0.60 & 0.89 \\
& HD 1 & 0.94 & 0.97 \\
& FTAI 2 & 0.59 & 0.94 \\
FSTC & HD 2 & 0.95 & 0.99 \\
& FTAI 0 & 0.18 & 0.06 \\
& HD 0 & 0.93 & 1.00 \\
& FTAI 1 & 0.59 & 0.96 \\
& HD 1 & 0.86 & 0.98 \\
& FTAI 2 & 0.57 & 0.96 \\
DO & HD 2 & 0.87 & 0.99 \\
& FTAI 1 & 0.71 & 0.93 \\
& HD 1 & 0.91 & 0.98 \\
& FTAI 2 & 0.69 & 0.96 \\
& HD 2 & 0.90 & 0.99
\end{tabular}

${ }^{1}$ CTFS $=$ calving to first service $(\mathrm{d}) ;$ FSTC $=$ first service to conception $(\mathrm{d}) ; \mathrm{DO}=$ days open $(\mathrm{d}) ; 0,1$, and $2=$ parity number. the original herd factor and was only run for the control scenario, as both the HD and FTAI groups only had one breeding method and therefore would produce the same results. Rank correlations for the updated model are presented in Table 7 . It is evident that even with the addition of breeding method to the model, there were only minor changes in the rank correlations between the control and both the HD and FTAI groups. The limited improvement in rank correlations by adding breeding method to the model suggests that breeding method likely has a genetic component, further suggesting that FTAI and HD are 2 different traits. This means adding the breeding method to the model will not account for the bias caused by the TAI records, and therefore more complex methods to account for the bias need to be investigated. One possible method would be to add highly genetically correlated traits into a multitrait model. In a simulation study, Oliveira Junior et al. (2021) reported that by using the traits angularity and BCS, the bias added by TAI to fertility phenotypes could be mitigated. In this case, animals using TAI would have their phenotype for fertility traits set to missing and a 5-trait animal model would be used to estimate their EBV. However, this was a simulation study, and a similar analysis is required with real data.

\section{Standardized Recording}

Throughout this study, it became evident that a standardized way of recording breeding information is required. In total, 2,046 unique breeding codes were classified into 2 groups in 3 different scenario groups. The lack of specificity and uniformity of the breeding codes has likely resulted in mistakes made along this process. When looking at specific techniques, numerous codes were used to define them; for example, there were 156 unique breeding codes for Ovsynch.

Another issue was the specificity within the breeding codes; for example, the word "natural" was the most common protocol in the entire data set with nearly half a million records and 75 unique breeding codes. However, this may include any number of conventional heat detection techniques as there was no information on which specific technique was used in these records. Therefore, a standardized collection method for these data must be put in place so that breeding programs and researchers can have higher quality data for their analysis.

\section{CONCLUSIONS}

The results of this study suggested that the use of TAI affects the accuracy of genetic evaluations for fertility traits in dairy cattle. In all traits analyzed, large 
reranking was observed when bulls were compared based on TAI records against heat detection records. Bulls reranking was much lower when the control scenario was compared against the heat detection scenario groups. However, when the top 100 bulls in the control scenario were looked at directly, large reranking occurred, which better represents the expected correlations that would be seen if this work was undertaken on any national selection index. In an attempt to account for the bias on EBVs, breeding method was added to the model. However, there were only minor changes in the rank correlations between scenario groups, further indicating the potential genetic differences between the traits. Though the results of this study seem to suggest a bias within genetic evaluations of fertility traits with the inclusion of TAI records, additional research is required to confirm the findings of this study. Future studies should look at high-reliability bulls specifically to determine if the levels of reranking remain. Also, future studies should aim to understand the potential genetic differences between the fertility traits split via management technology, possibly in a multiple trait analysis. Methods to account for the potential bias created by TAI should also be investigated, and for this, the method by which these data are collected needs to be improved, through creating a standardized way of recording breeding codes.

\section{ACKNOWLEDGMENTS}

This research was financially supported by Agriculture and Agri-Food Canada (Ottawa, Canada), and by additional contributions from Dairy Farmers of Canada (Ottawa, Canada), the Lactanet (Guelph, Canada), and the Canadian Dairy Commission (Ottawa, Canada) under the Agri-Science Clusters Initiative. As per the research agreement, aside from providing financial support, the funders have no role in the design and conduct of the studies, data collection and analysis or interpretation of the data. Researchers maintain independence in conducting their studies, own their data, and report the outcomes regardless of the results. The decision to publish the findings rests solely with the researchers. C. Baes acknowledges support from the Natural Sciences and Engineering Research Council of Canada, Canada Research Chair program. The authors have not stated any conflicts of interest.

\section{REFERENCES}

Bisinotto, R. S., and J. E. P. Santos. 2011. The use of endocrine treatments to improve pregnancy rates in cattle. Reprod. Fertil. Dev. 24:258-266. https://doi.org/10.1071/RD11916.
Bouquet, A., and J. Juga. 2013. Integrating genomic selection into dairy cattle breeding programmes: A review. Animal 7:705-713. https://doi.org/10.1017/S1751731112002248.

Caraviello, D. Z., K. A. Weigel, P. M. Fricke, M. C. Wiltbank, M. J. Florent, N. B. Cook, K. V. Nordlund, N. R. Zwald, and C. L. Rawson. 2006. Survey of management practices on reproductive performance of dairy cattle on large US commercial farms. J. Dairy Sci. 89:4723-4735. https://doi.org/10.3168/jds.S0022 -0302(06)72522-X.

Cerri, R. L. A., J. E. P. Santos, S. O. Juchem, K. N. Galvão, and R. C. Chebel. 2004. Timed artificial insemination with estradiol cypionate or insemination at estrus in high-producing dairy cows. J. Dairy Sci. 87:3704-3715. https://doi.org/10.3168/jds.S0022 $-0302(04) 73509-2$.

Denis-Robichaud, J., R. L. A. Cerri, A. Jones-Bitton, and S. J. LeBlanc. 2016. Survey of reproduction management on Canadian dairy farms. J. Dairy Sci. 99:9339-9351. https://doi.org/10.3168/jds .2016-11445.

Fleming, A., E. A. Abdalla, C. Maltecca, and C. F. Baes. 2018. Invited review: Reproductive and genomic technologies to optimize breeding strategies for genetic progress in dairy cattle. Arch. Tierzucht 61:43-57. https://doi.org/10.5194/aab-61-43-2018.

Gilmour, A. R., B. J. Gogel, B. R. Cullis, S. J. Welham, and R. Thompson. 2014. ASReml User Guide Release 4.1 Functional Specification. VSN Int. Ltd. www.vsni.co.uk.

Giordano, J. O., P. M. Fricke, J. N. Guenther, G. Lopes Jr., M. M. Herlihy, A. B. Nascimento, and M. C. Wiltbank. 2012. Effect of progesterone on magnitude of the luteinizing hormone surge induced by two different doses of gonadotropin-releasing hormone in lactating dairy cows. J. Dairy Sci. 95:3781-3793. https://doi.org/ $10.3168 /$ jds.2011-5155.

Goodling, R. C. Jr., G. E. Shook, K. A. Weigel, and N. R. Zwald. 2005. The effect of synchronization on genetic parameters of reproductive traits in dairy cattle. J. Dairy Sci. 88:2217-2225. https://doi .org/10.3168/jds.S0022-0302(05)72897-6.

Goodwin, L. D., and N. L. Leech. 2006. Understanding correlation: Factors that affect the size of r. J. Exp. Educ. 74:249-266. https:/ /doi.org/10.3200/JEXE.74.3.249-266.

Jamrozik, J., J. Fatehi, G. J. Kistemaker, and L. R. Schaeffer. 2005. Estimates of genetic parameters for Canadian Holstein female reproduction traits. J. Dairy Sci. 88:2199-2208. https://doi.org/10 .3168/jds.S0022-0302(05)72895-2.

Kadarmideen, H. N., R. Thompson, M. P. Coffey, and M. A. Kossaibati. 2003. Genetic parameters and evaluations from single- and multiple-trait analysis of dairy cow fertility and milk production. Livest. Prod. Sci. 81:183-195. https://doi.org/10.1016/S0301 -6226(02)00274-9.

Karakaya-Bilen, E., E. S. Ribeiro, R. S. Bisinotto, A. Gümen, and J. E. P. Santos. 2019. Effect of presynchronization with prostaglandin F $2 \alpha$ before the 5-d timed AI protocol on ovarian responses and pregnancy in dairy heifers. Theriogenology 132:138-143. https:// doi.org/10.1016/j.theriogenology.2019.03.019.

Leblanc, S. 2001. The Ovsynch breeding program for dairy cows - A review and economic perspective. Bov. Pract. 35:13-22.

Lima, F. S., E. S. Ribeiro, R. S. Bisinotto, L. F. Greco, N. Martinez, M. Amstalden, W. W. Thatcher, and J. E. P. Santos. 2013. Hormonal manipulations in the 5-day timed artificial insemination protocol to optimize estrous cycle synchrony and fertility in dairy heifers. J. Dairy Sci. 96:7054-7065. https://doi.org/10.3168/jds .2013-7093.

Lopez, H., L. D. Satter, and M. C. Wiltbank. 2004. Relationship between level of milk production and estrous behavior of lactating dairy cows. Anim. Reprod. Sci. 81:209-223. https://doi.org/10 .1016/j.anireprosci.2003.10.009.

Lucy, M. C., J. S. Stevenson, and E. P. Call. 1986. Controlling first service and calving interval by prostaglandin $\mathrm{F} 2 \alpha$, gonadotropinreleasing hormone, and timed insemination. J. Dairy Sci. 69:21862194. https://doi.org/10.3168/jds.S0022-0302(86)80652-X.

Madureira, A. M. L., T. A. Burnett, S. Borchardt, W. Heuwieser, C. F. Baes, J. L. M. Vasconcelos, and R. L. S. Cerri. 2021. Plasma concentrations of progesterone in the preceding estrous cycle are 
associated with the intensity of estrus and fertility of Holstein cows. PLoS One 16:e0248453. https://doi.org/10.1371/journal .pone. 0248453

Madureira, A. M. L., B. F. Silper, T. A. Burnett, L. Polsky, L. H. Cruppe, D. M. Veira, J. L. M. Vasconcelos, and R. L. A. Cerri. 2015. Factors affecting expression of estrus measured by activity monitors and conception risk of lactating dairy cows. J. Dairy Sci. 98:7003-7014. https://doi.org/10.3168/jds.2015-9672.

Moreira, F., C. Orlandi, C. A. Risco, R. Mattos, F. Lopes, and W. W. Thatcher. 2001. Effects of presynchronization and bovine somatotropin on pregnancy rates to a timed artificial insemination protocol in lactating dairy cows. J. Dairy Sci. 84:1646-1659. https: //doi.org/10.3168/jds.S0022-0302(01)74600-0.

Oliveira Junior, G. A., L. R. Schaeffer, F. Schenkel, F. Tiezzi, and C. F. Baes. 2021. Potential effects of hormonal synchronized breeding on genetic evaluations of fertility traits in dairy cattle: A simulation study. J. Dairy Sci. 104:4404-4412. https://doi.org/10.3168/ jds.2020-18944.

Pritchard, T., M. Coffey, R. Mrode, and E. Wall. 2013. Genetic parameters for production, health, fertility and longevity traits in dairy cows. Animal 7:34-46. https://doi.org/10.1017/ S1751731112001401.

Pursley, J. R., M. R. Kosorok, and M. C. Wiltbank. 1997. Reproductive management of lactating dairy cows using synchronization of ovulation. J. Dairy Sci. 80:301-306. https://doi.org/10.3168/jds .S0022-0302(97)75938-1.

R Core Development Team. 2019. R: A language and environment for statistical computing. R Foundation for Statistical Computing.

Rabaglino, M. B., C. A. Risco, M. J. Thatcher, I. H. Kim, J. E. P. Santos, and W. W. Thatcher. 2010. Application of one injection of prostaglandin F2 $\alpha$ in the five-day Co-Synch+CIDR protocol for estrous synchronization and resynchronization of dairy heifers. J. Dairy Sci. 93:1050-1058. https://doi.org/10.3168/jds.2009-2675.

Ribeiro, E. S., R. L. A. Cerri, R. S. Bisinotto, F. S. Lima, F. T. Silvestre, L. F. Greco, W. W. Thatcher, and J. E. P. Santos. 2011. Reproductive performance of grazing dairy cows following presynchronization and resynchronization protocols. J. Dairy Sci. 94:4984-4996. https://doi.org/10.3168/jds.2011-4225.

Ribeiro, E. S., K. N. Galvão, W. W. Thatcher, and J. E. P. Santos. 2012. Economic aspects of applying reproductive technologies to dairy herds. Anim. Reprod. 9:370-387.

Rivera, H., H. Lopez, and P. M. Fricke. 2005. Use of intravaginal progesterone-releasing inserts in a synchronization protocol before timed $\mathrm{AI}$ and for synchronizing return to estrus in Holstein heifers. J. Dairy Sci. 88:957-968. https://doi.org/10.3168/jds.S0022 -0302(05)72763-6.

Roelofs, J., F. López-Gatius, R. H. F. Hunter, F. J. C. M. van Eerdenburg, and C. Hanzen. 2010. When is a cow in estrus? Clinical and practical aspects. Theriogenology 74:327-344. https://doi.org/10 .1016/j.theriogenology.2010.02.016.

Roso, V. M., F. S. Schenkel, and S. P. Miller. 2004. Degree of connectedness among groups of centrally tested beef bulls. Can. J. Anim. Sci. 84:37-47. https://doi.org/10.4141/A02-094.
Santos, J. E. P., H. M. Rutigliano, and M. F. S. Filho. 2009. Risk factors for resumption of postpartum estrous cycles and embryonic survival in lactating dairy cows. Anim. Reprod. Sci. 110:207-221. https://doi.org/10.1016/j.anireprosci.2008.01.014.

Santos, V. G., P. D. Carvalho, C. Maia, B. Carneiro, A. Valenza, P. M. Crump, and P. M. Fricke. 2016. Adding a second prostaglandin F2 $\alpha$ treatment to but not reducing the duration of a PRID-Synch protocol increases fertility after resynchronization of ovulation in lactating Holstein cows. J. Dairy Sci. 99:3869-3879. https://doi .org/10.3168/jds.2015-10557.

Santos, V. G., P. D. Carvalho, C. Maia, B. Carneiro, A. Valenza, and P. M. Fricke. 2017. Fertility of lactating Holstein cows submitted to a Double-Ovsynch protocol and timed artificial insemination versus artificial insemination after synchronization of estrus at a similar day in milk range. J. Dairy Sci. 100:8507-8517. https://doi .org/10.3168/jds.2017-13210.

Sartori, R., J. M. Haughian, R. D. Shaver, G. J. M. Rosa, and M. C. Wiltbank. 2004. Comparison of ovarian function and circulating steroids in estrous cycles of Holstein heifers and lactating cows. J. Dairy Sci. 87:905-920. https://doi.org/10.3168/jds.S0022 -0302(04)73235-X.

Silper, B. F., A. M. L. Madureira, L. B. Polsky, S. Soriano, A. F Sica, J. L. M. Vasconcelos, and R. L. A. Cerri. 2017. Daily lying behavior of lactating Holstein cows during an estrus synchronization protocol and its associations with fertility. J. Dairy Sci. 100:8484-8495. https://doi.org/10.3168/jds.2016-12160.

Souza, A. H., H. Ayres, R. M. Ferreira, and M. C. Wiltbank. 2008. A new presynchronization system (Double-Ovsynch) increases fertility at first postpartum timed AI in lactating dairy cows. Theriogenology 70:208-215. https://doi.org/10.1016/j.theriogenology 2008.03.014.

Tenhagen, B. A., M. Drillich, R. Surholt, and W. Heuwieser. 2004 Comparison of timed AI after synchronized ovulation to AI at estrus: Reproductive and economic considerations. J. Dairy Sci. 87:85-94. https://doi.org/10.3168/jds.S0022-0302(04)73145-8.

Tsuruta, S., J. F. Keown, L. D. Van Vleck, and I. Misztal. 2000. Bias in genetic evaluations by records of cows treated with bovine somatotropin. J. Dairy Sci. 83:2650-2656. https://doi.org/10.3168/ jds.S0022-0302(00)75158-7.

Vailes, L. D., and J. H. Britt. 1990. Influence of footing surface on mounting and other sexual behaviors of estrual Holstein cows. J. Anim. Sci. 68:2333. https://doi.org/10.2527/1990.6882333x.

Van Doormaal, B. 2018. The Fertility Challenge. Accessed May 5, 2019. https://www.cdn.ca/document.php?id=493.

Van Vleck, L. D. 1993. Variance of prediction error with mixed model equations when relationships are ignored. Theor. Appl. Genet. 85:545-549. https://doi.org/10.1007/BF00220912.

Wiltbank, M., H. Lopez, R. Sartori, S. Sangsritavong, and A. Gümen. 2006. Changes in reproductive physiology of lactating dairy cows due to elevated steroid metabolism. Theriogenology 65:17-29. https://doi.org/10.1016/j.theriogenology.2005.10.003. 